\title{
WNT1-inducible signaling pathway protein-1 contributes to tumor progression and treatment failure in oral squamous cell carcinoma
}

\author{
EUN KYUNG JUNG ${ }^{1 *}$, SUN-AE KIM ${ }^{1 *}$, TAE MI YOON ${ }^{1}$, KYUNG-HWA LEE $^{2}$, HEE KYUNG KIM $^{3}$, \\ DONG HOON LEE ${ }^{1}$, JOON KYOO LEE ${ }^{1}$, IK-JOO CHUNG ${ }^{3}$, YOUNG-EUN JOO ${ }^{3}$ and SANG CHUL LIM ${ }^{1}$ \\ Departments of ${ }^{1}$ Otorhinolaryngology-Head and Neck Surgery, ${ }^{2}$ Pathology and ${ }^{3}$ Internal Medicine, \\ Chonnam National University Medical School, Gwangju, Chonnam 58128, Republic of Korea
}

Received January 10, 2017; Accepted April 3, 2017

DOI: $10.3892 / \mathrm{ol} .2017 .6313$

\begin{abstract}
WNT1-inducible-signaling pathway protein-1 (WISP-1) belongs to the family of cysteine rich 61/connective tissue growth factor/nephroblastoma overexpressed matricellular proteins, which are involved in various biological processes, including cell adhesion, proliferation, differentiation, angiogenesis and carcinogenesis. In the present study, the expression of WISP-1 was investigated, and its association with clinicopathological factors and prognosis in patients with oral squamous cell carcinoma (OSCC) was evaluated. Additionally, the role of WISP-1 in invasion and apoptosis of human OSCC cells was evaluated. Immunoreactivity of WISP-1 was increased in OSCC tissue compared with adjacent normal tissue samples. High expression of WISP-1 protein was observed in 24/84 (28.57\%) OSCC specimens. Additionally, high WISP-1 expression was significantly associated with treatment failure $(\mathrm{P}=0.042)$. The 5-year overall survival rate was $33 \%$ in patients with high WISP1 expression, and $66 \%$ in patients with low WISP-1 expression. WISP-1 expression in the human OSCC SCC-1483 cell line was observed. Furthermore, WISP-1 knockdown using small interfering (si)RNA significantly reduced cell invasion and induced apoptosis compared with control siRNA-transfected cells. These findings suggested that WISP-1 is associated with tumor progression and poor prognosis by increasing tumor cell invasion and inhibiting cell apoptosis in human OSCC.
\end{abstract}

Correspondence to: $\mathrm{Dr}$ Tae Mi Yoon, Department of Otorhinolaryngology-Head and Neck Surgery, Chonnam National University Medical School, 264 Seoyangro, Gwangju, Chonnam 58128, Republic of Korea

E-mail: yoontm@chonnam.ac.kr

${ }^{*}$ Contributed equally

Key words: WNT1-inducible-signaling pathway protein-1, mouth neoplasms, squamous cell carcinoma, molecular targeted therapy, disease progression, treatment failure, survival

\section{Introduction}

Oral squamous cell carcinoma (OSCC) is the most type of common head and neck cancer, representing 1-2\% of all human malignancies (1). Despite advancements in diagnosis and treatment strategies in recent decades, OSCC remains associated with a poor prognosis and low survival rates (1). The major characteristics of cancer cells, including the ability to invade and metastasize, are associated with tumor progression, and ultimately with patient survival. Identifying molecular changes in cancer has been a research focus worldwide, with the aim of developing potential therapeutic methods. Therefore, understanding the molecular and biological characteristics of OSCC is essential for the development of more efficacious therapies.

WNT1-inducible-signaling pathway protein-1 (WISP-1) belongs to the cysteine rich 61 (Cyr61)/connective tissue growth factor (CTGF)/nephroblastoma overexpressed (Nov) $(\mathrm{CCN})$ family of matricellular proteins, which are secreted proteins associated with the extracellular matrix (ECM) (2-4). The ECM modulates cellular responses, including cell growth, differentiation and survival (2-4). Since the first identification of human WISP-1 in an epithelial cell line in 1998, WISP-1 expression has been studied in various organs and disease states, including malignant diseases, such as colorectal cancer, hepatocellular carcinoma, lung carcinoma and breast cancer, and nonmalignant diseases, including lung fibrosis (5-10). WISP-1 is strongly expressed in primary breast cancer and rectal cancer, and increased WISP-1 expression is associated with more aggressive features of cancer progression $(8,10)$. Although WISP-1 has been the focus of numerous studies in identifying novel potential molecular therapeutic targets, the function of WISP-1 in human OSCC remains unclear.

In the present study, WISP-1 expression in OSCC was examined, and the association between its expression and clinicopathological characteristics was evaluated in a well-defined series of human OSCC samples. Furthermore, its prognostic value was evaluated in OSCC. To the best of our knowledge, the present study is the first to demonstrate the prognostic value of WISP-1 in OSCC. In addition, the contribution of WISP-1 to cell invasion and apoptosis, which are associated with tumor progression, and survival, was analyzed in human OSCC cells. By considering the clinicopathological characteristics and molecular findings, the results of the present study 
may provide the basis for the mechanism of WISP-1 function in human OSCC.

\section{Materials and methods}

Patients and tumor specimens. Formalin-fixed paraffinembedded tumor and normal stroma tissue sections were collected from 95 patients who underwent surgical treatment for either diagnostic biopsy or definitive surgery for OSCC at Chonnam National University Hwasun Hospital (Gwangju, Korea) between June 2013 and May 2004. A total of 61 men and 23 women participated in the present study with a mean age of $63.03 \pm 12.51$ years (range, 26-87 years). A total of 11 patients were excluded due to failure to follow-up, palliative intent, or loss of paraffin-embedded tissue blocks. WISP-1 expression and clinicopathological parameters, including age, sex, tumor location, overall, tumor (T) and node (N) stages, recurrence, treatment failure and adjuvant treatment, were analyzed using the collected OSCC tissue samples. Following primary treatment with curative intent, patients who exhibited locoregional recurrence underwent salvage surgery or concurrent chemoradiotherapy. Those with inoperable locoregional progression or distant metastasis, even following salvage treatment, were defined as treatment failure. Tumor staging was performed according to the seventh edition of the American Joint Committee on Cancer Staging system (11). Survival was calculated using the date of starting treatment and the date of mortality or last follow-up. The present study was approved by the Ethics Committee of the Institutional Review Board of Chonnam National University Hwasun Hospital. Written informed consent was obtained from each patient prior to tissue acquisition.

Immunohistochemistry. Tissue sections (thickness, $5 \mu \mathrm{m}$ ) were cut from the paraffin blocks of OSCC tissue, and sections were mounted and dried on glass slides. Tissues were deparaffinized and rehydrated using a graded series of ethanol (100, $95,90,80,70$ and 60\%), and antigen retrieval was performed using 1X Citrate buffer (cat. no. S2031; Dako; Agilent Technologies, Inc., Santa Clara, CA, USA) for 10 min using a steam cooker. Endogenous peroxidase activity was blocked using peroxidase-blocking solution (Dako; Agilent Technologies, Inc.) for $10 \mathrm{~min}$ at room temperature, followed by incubation with polyclonal rabbit anti-human antibody directed against WISP-1 (cat. no. sc-25441; 1:100; Santa Cruz Biotechnology, Inc., Dallas, TX, USA) overnight at $4^{\circ} \mathrm{C}$. Tissues were incubated with Polymer horseradish peroxidase (HRP) antirabbit and antimouse IgG (cat. no. D11-110; Ready-to-use; Golden Bridge International, Inc., Bothwell, WA, USA) for $10 \mathrm{~min}$ at room temperature. Following washing in TBS-Tween 20 buffer (Biosesang, Sungnam, Korea), tissues were stained using a DAB 20X HRP detection kit (cat. no. C09-12; Golden Bridge International, Inc.) for $3 \mathrm{~min}$ at room temperature. Tissues were counterstained with hematoxylin and mounted with coverslips. Stained tissues were observed and imaged using a light microscope. Assessment of staining was interpreted by two independent observers who were blinded to the clinical information. Assessment of staining intensity was performed as follows: 0, no staining of tumor cells; $1+$, weak to comparable staining in the cytoplasm and/or nucleus compared with that of non-tumor cells; $2+$, readily appreciable or dark brown staining distinctly marking the tumor cell cytoplasm and/or nucleus. Specimens with 0 or $1+$ staining were regarded as low expression and those with $2+$ staining were regarded as high expression.

Cell culture and transfection. The human OSCC SCC-1483 cell line was provided by Dr. Kim CH (Ajou University, Suwon, Korea). The cells were cultured in RPMI-1640 medium supplemented with $10 \%$ fetal bovine serum (both from HyClone; GE Healthcare Life Sciences, Logan, UT, USA), $50 \mathrm{U} / \mathrm{ml}$ penicillin and $50 \mu \mathrm{g} / \mathrm{ml}$ streptomycin (Gibco; Thermo Fisher Scientific, Inc., Waltham, MA, USA) in a humidified atmosphere containing $5 \% \mathrm{CO}_{2}$ at $37^{\circ} \mathrm{C}$. SCC-1483 cells were seeded into 6 -well plates at a density of $2 \times 10^{5}$ cells/well and transfected with 100 pmol WISP-1-specific small interfering (si)RNA (cat. no., 1164842; Bioneer Corporation, Daejeon, Korea) or negative control siRNA (AllStar Neg. Control; cat. no., 1027281; Qiagen, Inc., Valencia, CA, USA) using Lipofectamine RNA iMAX (Invitrogen; Thermo Fisher Scientific, Inc.) for $48 \mathrm{~h}$ at $37^{\circ} \mathrm{C}$.

RNA isolation and reverse transcription polymerase chain reaction ( $R T-P C R)$. Total RNA was isolated using TRIzol reagent (Invitrogen; Thermo Fisher Scientific, Inc.) according to the manufacturer's protocol. Total RNA $(1 \mu \mathrm{g})$ was mixed with $1 \mu 110 \mathrm{mM}$ dNTP mix (Enzynomics, Daejeon, Korea), $1 \mu \mathrm{l}$ oligo dT $(500 \mu \mathrm{g} / \mathrm{ml}$; Promega Corporation, Madison, WI, USA), $2 \mu 1$ DTT (0.1 M), $4 \mu 1$ 5X First-strand buffer, $2 \mu \mathrm{l}$ M-MLV reverse transcriptase (both Invitrogen; Thermo Fisher Scientific, Inc.) and $1 \mu 1$ RNase inhibitor (Promega Corporation). Gene-specific primers were subsequently used to amplify the complementary DNA using PCR. A total of $0.1 \mu \mathrm{l}$ GoTaq DNA polymerase and $4 \mu \mathrm{l} 5 \mathrm{X}$ Green GoTaq DNA polymerase reaction buffer (both from Promega Corporation) were used for amplification. The primer sequences were as follows: WISP-1 forward, 5'-CTCAGCAGCTTGGGGACA AC-3' and reverse, 5'-GATGCCTCTGGCTGGTACAC-3'; and glyceraldehyde-3-phosphate dehydrogenase $(G A P D H)$ forward, 5'-ACCACAGTCCATGCCATCAC-3' and reverse primer, 5'-TCCACCACCCTGTTGCTGTA-3' (Bioneer Corporation). WISP-1 expression was evaluated using the following thermocycling conditions: $94^{\circ} \mathrm{C}$ for $5 \mathrm{~min} ; 32$ cycles of $94^{\circ} \mathrm{C}$ for $30 \mathrm{sec}, 55^{\circ} \mathrm{C}$ for $30 \mathrm{sec}, 72^{\circ} \mathrm{C}$ for $30 \mathrm{sec}$ and a final elongation step at $72^{\circ} \mathrm{C}$ for $7 \mathrm{~min}$. Following separating PCR products using electrophoresis on $1 \%$ agarose gels (Lonza, Rockland, ME, USA) containing ethidium bromide (Bioneer Corporation). Multi Gauge software (version 3.2; Fujifilm, Tokyo, Japan) was used to quantify the signal using densitometric analysis.

Protein isolation and western blot analysis. The cells and tissue samples were lysed in RIPA buffer (1 M Tris- $\mathrm{HCl}$, $150 \mathrm{mM} \mathrm{NaCl}, 1 \%$ Triton X-100, 2 mM EDTA) with $1 \mathrm{mM}$ phenylmethanesulfonyl fluoride, $1 \mathrm{mM}$ Halt phosphatase inhibitor and $1 \mathrm{mM}$ Halt protease inhibitor cocktail(Thermo Fisher Scientific, Inc.). The resolved protein concentrations were measured using a BCA kit, and 20-30 $\mu \mathrm{g}$ protein/lane was separated through SDS-PAGE on $10-12 \%$ gels and transferred to polyvinylidene fluoride membranes (EMD Millipore, Billerica, MA, USA). Following incubation of the membranes 
for $1 \mathrm{~h}$ at room temperature in blocking solution [5\% bovine serum albumin (BSA); BioShop, Inc., Burlington, ON, Canada] and washing four times for 15 min with TBS-Tween 20 buffer, specific proteins were sequentially blotted with primary antibodies. The following antibodies were used: Rabbit anti-human polyclonal antibody directed against WISP-1 (cat. no. sc-25441) and rabbit anti-human antibody directed against GAPDH (cat. no. sc-25778) (Santa Cruz Biotechnology, Inc.). Antibodies were diluted at 1:1,000 and incubated with the membranes for $24 \mathrm{~h}$ at $4^{\circ} \mathrm{C}$. An enhanced chemiluminescence detection system with $1 \mathrm{X}$ horseradish peroxidase substrate (cat. no. WBKL S00 50; EMD Millipore) was used to visualize immunoreactive proteins for $30 \mathrm{sec}$ at room temperature, and an LAS-4000 luminescent image analyzer (Fujifilm, Tokyo, Japan) was used to analyze the proteins.

Cell invasion assay. Cell invasion ability was calculated according to the total number of cells invading through a Matrigel-coating Transwell invasion apparatus with $8 \mu \mathrm{m}$ pores. The cells transfected with WISP-1 siRNA or negative control siRNA $\left(3 \times 10^{5}\right.$ cells/well) were seeded into the upper chambers with $120 \mu \mathrm{l}$ RPMI-1640 medium containing $0.2 \%$ BSA. Next, $400 \mu \mathrm{l} 0.2 \%$ BSA containing $7 \mu \mathrm{g} / \mathrm{ml}$ fibronectin (EMD Millipore) was added to the lower chamber as the chemoattractant. Following incubation for $24 \mathrm{~h}$ at room temperature, Diff Quik solution (Sysmex Corporation, Kobe, Japan) was used to stain the bottom Transwell surface containing the invaded cells. Five random squares in the light microscopic field of view were measured, and the results are presented as the means \pm standard error of the number of cells/field in three individual experiments.

Apoptosis assay. Annexin V-fluorescein isothiocyanate (FITC) assays were used to determine apoptotic rates. The cells transfected with WISP-1 siRNA or negative control siRNA were collected with trypsin for $5 \mathrm{~min}$ at room temperature $(179 \mathrm{x}$ g), washed twice in phosphate-buffered saline and resuspended in binding buffer (BD Biosciences, San Jose, CA, USA) following $48 \mathrm{~h}$. Subsequently, annexin V-FITC and 7-amino-actino mycin D (BD Biosciences) were added. The cells were incubated in the dark for $15 \mathrm{~min}$ at room temperature and resuspended in $400 \mu \mathrm{l}$ binding buffer. A FACSCalibur flow cytometer was used, and the data were analyzed with BD Cell Quest (version 3.3; BD Biosciences) and WinMDI (version 2.9; The Scripps Research Institute, San Diego, CA, USA).

Statistical analysis. Associations between WISP-1 expression and various clinicopathological parameters were compared using the $\chi^{2}$ test and Fisher's exact test. Survival curves were calculated using the Kaplan-Meier estimator method and compared using the log-rank test. Student's t-tests were used to assess the significance of experimental differences. Analyses were performed using SPSS software (version 21.0; IBM Corp., Armonk, NY, USA). $\mathrm{P}<0.05$ were considered to indicate a statistically significant difference.

\section{Results}

Immunoreactivity for WISP-1 is increased in OSCC tissues compared with adjacent normal tissues. Table I summarizes
Table I. Association between WISP-1 expression and clinicopathological parameters in patients with oral squamous cell carcinoma $(\mathrm{n}=84)$.

WISP-1 expression

\begin{tabular}{|c|c|c|c|}
\hline \multirow[b]{2}{*}{ Parameters } & \multicolumn{2}{|c|}{ WISP-1 expression } & \multirow[b]{2}{*}{ P-value } \\
\hline & Low $(n=60)$ & High $(n=24)$ & \\
\hline Age, years & & & 0.890 \\
\hline$<63.03$ & 29 & 12 & \\
\hline$\geq 63.03$ & 31 & 12 & \\
\hline Sex & & & 0.757 \\
\hline Male & 43 & 18 & \\
\hline Female & 17 & 6 & \\
\hline Location & & & 0.651 \\
\hline Oral tongue & 43 & 16 & \\
\hline FOM, BM or RMT & 17 & 8 & \\
\hline Stage & & & 0.885 \\
\hline I, II & 39 & 16 & \\
\hline III, IV & 21 & 8 & \\
\hline T stage & & & 0.413 \\
\hline $\mathrm{T} 1, \mathrm{~T} 2$ & 51 & 22 & \\
\hline $\mathrm{T} 3, \mathrm{~T} 4$ & 9 & 2 & \\
\hline $\mathrm{N}$ stage & & & 0.940 \\
\hline No & 42 & 17 & \\
\hline $\mathrm{N} 1, \mathrm{~N} 2$ & 18 & 7 & \\
\hline CRT & & & 1.000 \\
\hline No & 35 & 14 & \\
\hline Yes & 25 & 10 & \\
\hline Recurrence & & & 0.283 \\
\hline Negative & 40 & 13 & \\
\hline Positive & 20 & 11 & \\
\hline Treatment failure & & & 0.042 \\
\hline Negative & 46 & 13 & \\
\hline Positive & 14 & 11 & \\
\hline
\end{tabular}

FOM, floor of mouth; BM, buccal mucosa; RMT, retromolar trigone; CRT, chemotherapy and/or radiotherapy; T, tumor; N, node; WISP-1, WNT1-inducible-signaling pathway protein-1.

the clinicopathological characteristics of 84 patients with OSCC included in the present study group. WISP-1 protein expression was examined immunohistochemically in formalin-fixed paraffin-embedded blocks of specimens from patients with OSCC.WISP-1 immunoreactivity in OSCC cells demonstrated heterogeneous patterns with stronger immunostaining in the cytoplasm and/or nuclei compared with the cells in the normal stromal tissue (Fig. 1A). By contrast, minimal staining for WISP-1 immunoreactivity was observed in OSCC cells compared with normal stromal tissue as a negative control (Fig. 1B). These immunohistochemical analyses revealed that 24/84 (28.57\%) OSCC specimens exhibited high WISP-1 expression.

WISP-1 expression is associated with treatment failure and survival in OSCC. To elucidate the prognostic role 

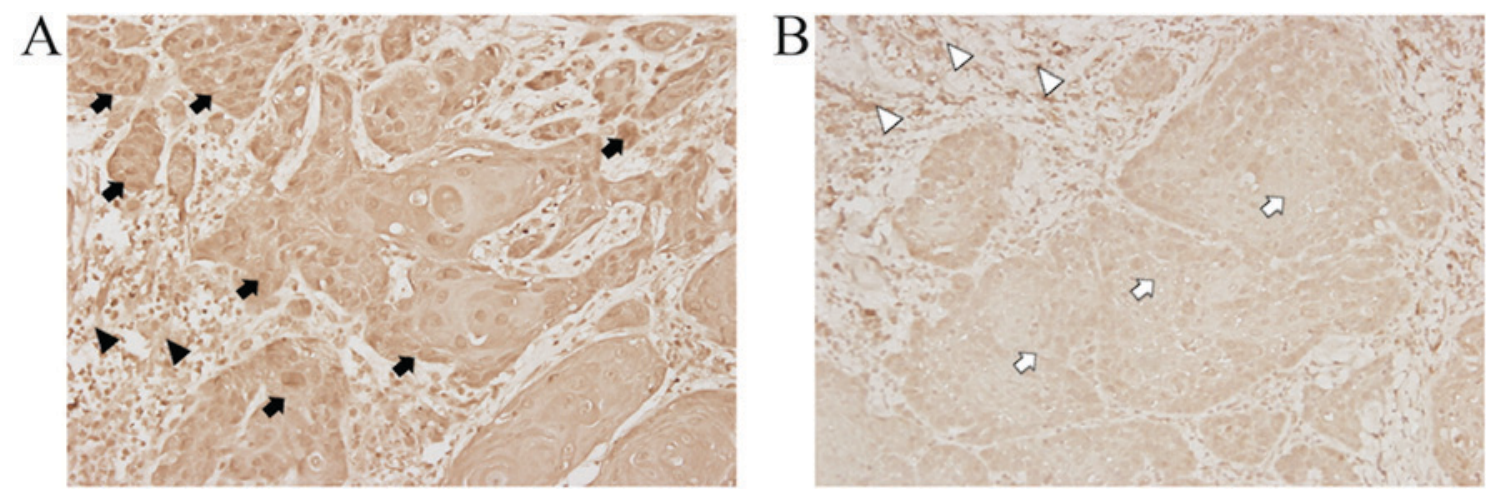

Figure 1. Comparison of WISP-1 protein expression in OSCC tissue samples and adjacent normal tissues. (A) Representative image demonstrating WISP-1 stain intensity score of $2+$ (high expression) with appreciable brown staining of OSCC cells (black arrow) compared with adjacent normal stromal tissue (black arrow head). (B) Representative image demonstrating relatively minimal staining for WISP-1 immunoreativity (1+ intensity score, low expression) with weak staining of OSCC cells (white arrow) compared with normal stromal tissue (white arrow head). Magnification, x400. OSCC, oral squamous cell carcinoma; WISP-1, WNT1-inducible-signaling pathway protein-1.

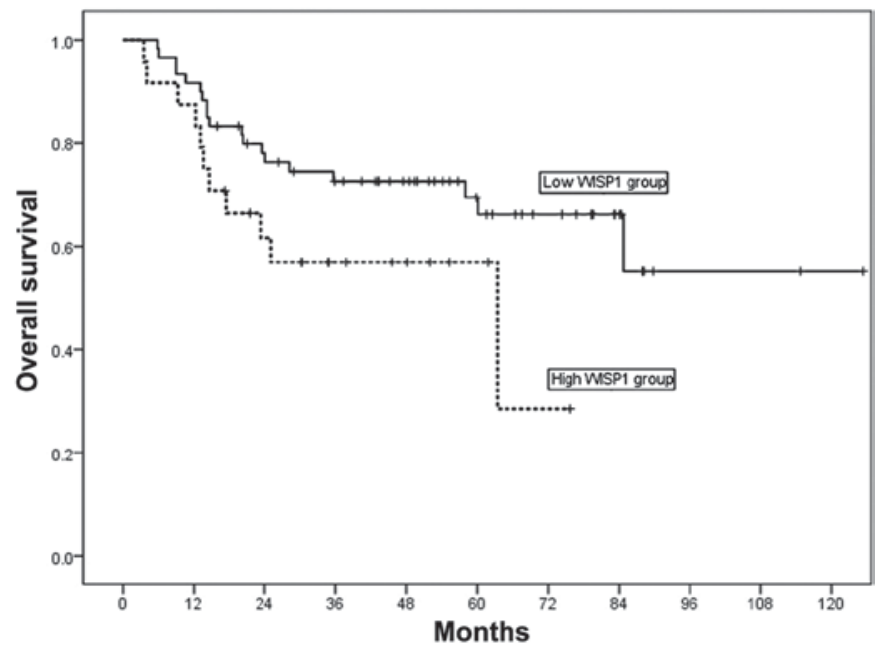

Figure 2. Kaplan-Meier curves demonstrating OS in patients with OSCC according to WISP-1 expression. High WISP-1 expression tended to decrease OS in patients with OSCC, however log-rank tests did not demonstrate a significant difference between these patients with high and low expression of WISP-1 ( $\mathrm{P}=0.075$ vs. low expression). Solid line, patients with low WISP-1 expression; dotted line, patient with high WISP-1 expression. OS, overall survival; OSCC, oral squamous cell carcinoma; WISP-1, WNT1-inducible-signaling pathway protein-1.

of WISP-1 protein in OSCC, the associations between WISP-1 expression and clinicopathological parameters were analyzed. WISP-1 expression in OSCC was not significantly associated with age, sex, primary tumor location, overall stage, T stage, $\mathrm{N}$ stage, chemoradiotherapy and recurrence (P>0.05; Table I). Treatment failure was identified to be significantly associated with WISP-1 expression $(\mathrm{P}=0.042$; Table I).

For the 84 patients enrolled in the present study, the 3- and 5-year overall survival (OS) rates were 68 and 59\%, respectively. The 5 -year OS rate was $33 \%$ in patients with high WISP-1 expression, and $66 \%$ in patients with low WISP1 expression (Fig. 2). High WISP-1 expression tended to decrease OS in patients with OSCC; however, analysis of Kaplan-Meier curves for OS using log-rank tests did not demonstrate a significant difference between these two groups of patients ( $\mathrm{P}=0.075$; Fig. 2$)$.
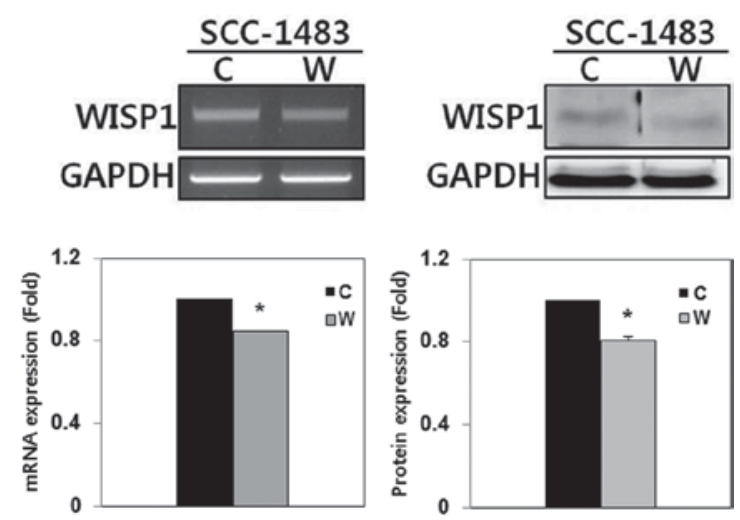

Figure 3. WISP-1 knockdown by siRNA in human OSCC cells. WISP-1 mRNA and protein were expressed in SCC-1483 OSCC cells. WISP-1 mRNA and protein expression were significantly reduced following transfection with WISP-1 siRNA in OSCC cells compared with the negative control. "P<0.05. GAPDH, glyceraldehyde 3-phosphate dehydrogenase; C, negative control siRNA-transfected cells; W, WISP-1-specific siRNA-transfected cells; WISP-1, WNT1-inducible-signaling pathway protein-1; siRNA, small interfering RNA; OSCC, oral squamous cell carcinoma.

WISP-1 knockdown suppresses invasion in OSCC cells. The expression of WISP-1 mRNA and protein was examined using RT-PCR and western blotting, respectively, in SCC-1483 cells. RT-PCR and western blotting demonstrated that WISP-1 was expressed in SCC-1483 cells (Fig. 3). To elucidate the function of WISP-1 in tumor progression in human OSCC cells, WISP-1 siRNA was used to suppress endogenous WISP-1 expression in SCC-1483 cells. WISP-1 mRNA and protein expression levels were reduced following WISP-1 siRNA transfection as compared with the cells transfected with negative control siRNA (Fig. 3).

The number of invaded cells in the WISP-1-knockdown group was $128.30 \pm 20.48$ compared with $271.80 \pm 15.50$ cells in the negative control group (Fig. 4). The difference between the two groups was statistically significant $(\mathrm{P}<0.05)$. Therefore, WISP-1 knockdown resulted in reduced cell invasiveness in human OSCC cells.

WISP-1 knockdown induces apoptosis in OSCC cells. Annexin V-FITC apoptosis assays were used to evaluate the 

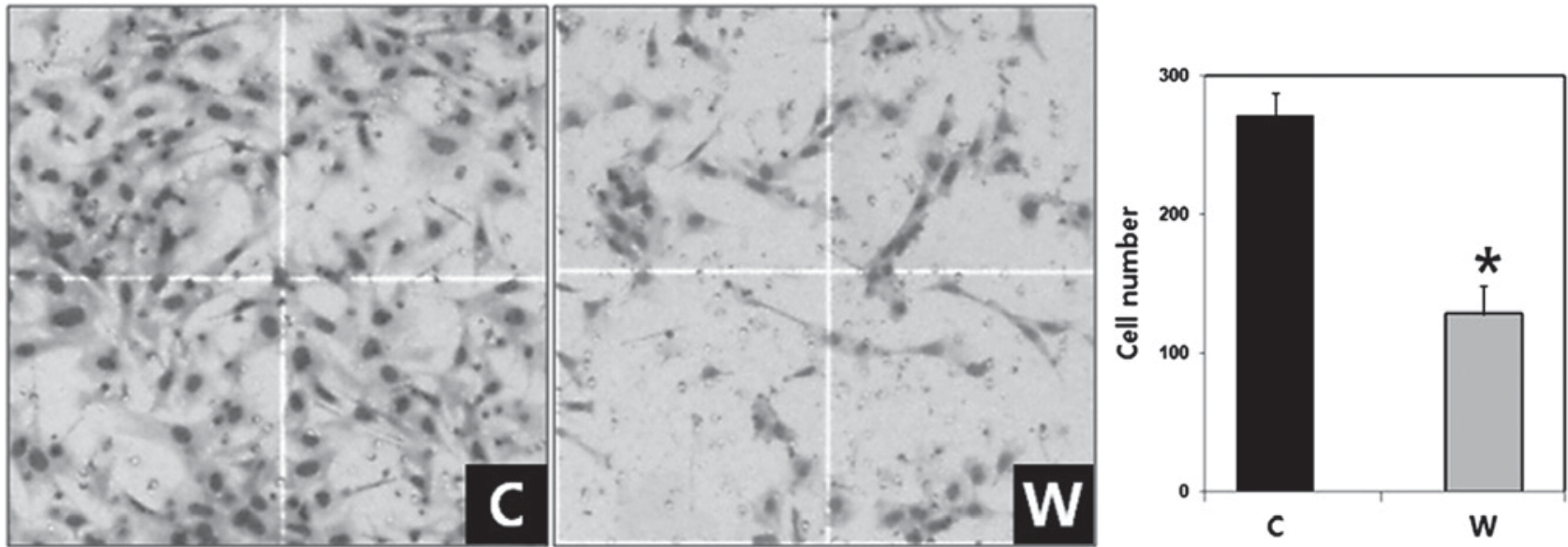

Figure 4. Effect of WISP-1 knockdown on invasion in human oral squamous cell carcinoma cells. Cell invasion assays revealed that WISP-1 knocked down cells exhibited significantly reduced invasion compared with the negative control cells. Stained invading cells were counted. Data are presented as the mean \pm standard error, and experiments were performed in triplicate. " $\mathrm{P}<0.05$. Magnification, $\mathrm{x} 40$. C, negative control siRNA-transfected cells; W, WISP-1-specific siRNA-transfected cells; WISP-1, WNT1-inducible-signaling pathway protein-1; siRNA, small interfering RNA.
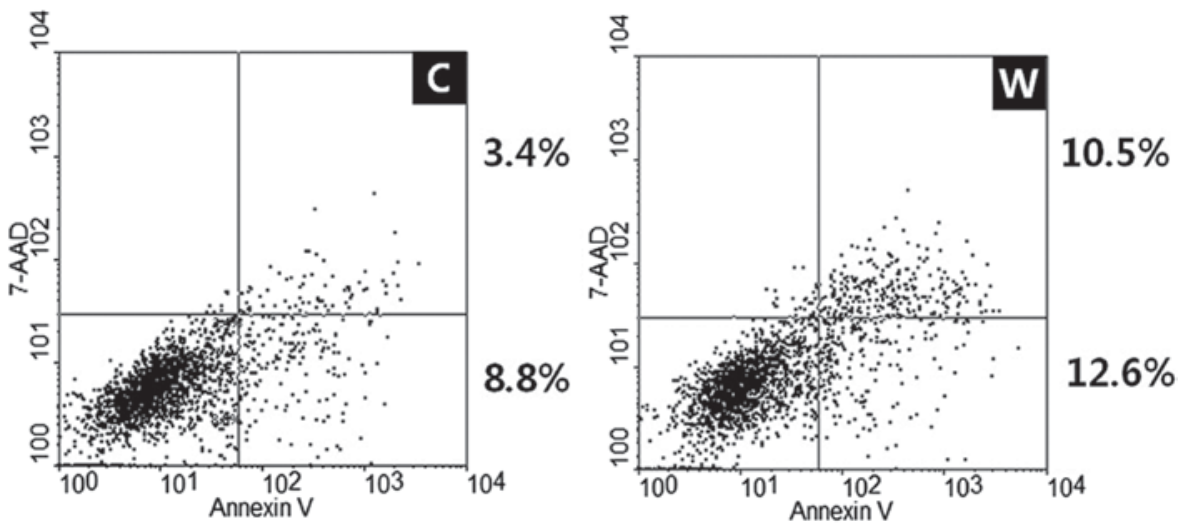

Figure 5. Effect of WISP-1 knockdown on cell apoptosis in human oral squamous cell carcinoma cells. The proportion of apoptotic cells observed for WISP-1 knocked down cells was greater compared with the negative control cells $(8.8+3.4 \mathrm{vs} .12 .6+10.5 \%)$. C, negative control siRNA-transfected cells; W, WISP-1-specific siRNA-transfected cells; WISP-1, WNT1-inducible-signaling pathway protein-1; siRNA, small interfering RNA; 7-AAD, 7-amino-actino mycin D.

effects of WISP-1 on cell apoptosis. Transfection with WISP-1 siRNA and negative control siRNA in SCC-1483 cells induced early and late apoptosis, with an increased apoptotic rate observed in cells transfected with WISP-1 siRNA compared with cells transfected with negative control siRNA (12.2 vs. $23.1 \%$, respectively; Fig. 5). Therefore, these results revealed that WISP-1 knockdown increased apoptosis in human OSCC cells.

\section{Discussion}

Identifying altered molecular mechanisms responsible for tumor invasion and metastasis is essential for developing potential therapeutic methods. By extension, identifying molecular markers that can detect cancer earlier or monitor cancer progression would enable personalization of medicine and improve the survival rate of patients with cancer (12). WISP-1 is a member of the CCN protein family (2-4). The CCN family includes CYR61 (CCN1), CTGF (CCN2), NOV (CCN3), WISP-1 (CCN4), WISP-2 (CCN5) and WISP-3 (CCN6) (2-4). The majority of $\mathrm{CCN}$ family proteins are secreted and expression of these proteins are associated with the ECM, which has been suggested to serve essential roles in tumorigenesis-associated processes, including tumor survival, proliferation, migration, and invasion (2). These findings have increased the interest towards investigating the potential of $\mathrm{CCN}$ proteins as therapeutic targets.

In adult human tissue, WISP-1 mRNA has been demonstrated to be expressed in number of organs, including the heart, kidney, lung, pancreas, placenta, ovary, small intestine and spleen (5). Notably, increased WISP-1 expression has been identified in several cancer types, including colorectal cancer, hepatocellular carcinoma, lung carcinoma, breast, esophageal and endometrial cancer (5-8,10,13-15). Furthermore, WISP-1 expression has also been revealed to be low in healthy lung epithelial cells, but significantly upregulated in lung cancer tissue (15).

In the present study, 24/84 (28.57\%) OSCC specimens demonstrated high WISP-1 expression. In addition, it was revealed that high WISP-1 expression was significantly associated with treatment failure. This finding is important as treatment failure leads to poor prognosis and survival in 
patients with OSCC. The 5-year OS rate was $33 \%$ in patients with high WISP-1 expression and 66\% in patients with low WISP-1 expression. Although the number of patients with OSCC in the current study was not sufficient to demonstrate a significant difference between the two groups, high WISP-1 expression tended to be associated with reduced survival rates in patients with OSCC. In esophageal and endometrial cancer, high WISP-1 expression has also been revealed to be associated with poor survival and clinicopathological parameters indicative of aggressive disease $(13,14)$. Furthermore, in a previous study, Chuang et al (16) reported that high WISP-1 expression was associated with an increased tumor stage in patients with OSCC. Taken together, these results and the findings of the present study suggest that WISP-1 expression may be used to predict poor clinical outcomes in patients with OSCC.

The dysregulation of cell invasion and apoptosis is a principle characteristic of cancer cells (17). OSCC is characterized by high rates of local invasion, and depth of invasion is a significant prognostic factor in patients with OSCC (18). Therefore, the role of WISP-1 in cell invasion and apoptosis was investigated using the human OSCC SCC-1483 cell line. siRNA-mediated WISP-1 knockdown significantly decreased cell invasion compared with siRNA-control transfected cells. In addition, WISP-1 knockdown induced apoptosis in SCC-1483 cells. Furthermore, a previous study reported that WISP-1 increased the invasive activity of SCC4 cells, another OSCC cell line (16). Additionally, You et al (19) and Su et al (20) demonstrated that WISP-1 suppresses c-myc-induced and p53-mediated apoptotic signaling pathways. These studies are consistent with the results of the present study and suggest that WISP-1 promotes aggressive phenotypes in OSCC cells.

In conclusion, although additional studies are warranted to support the findings of the present study, these results suggest that WISP-1 is associated with tumor progression and poor prognosis by increasing tumor cell invasion, and inhibiting cell apoptosis in human OSCC.

\section{Acknowledgements}

The present study was supported by the Chonnam National University Hwasun Hospital Institute of Biomedical Science (grant no. HCRI 16922-21). The authors would like to thank Dr CH Kim (Ajou University) for providing the SCC-1483 cell line.

\section{References}

1. Siegel R, Naishadham D and Jemal A: Cancer statistics, 2013. CA Cancer J Clin 63: 11-30, 2013.

2. Kleer CG, Zhang Y, Pan Q and Merajver SD: WISP3 (CCN6) is a secreted tumor-suppressor protein that modulates IGF signaling in inflammatory breast cancer. Neoplasia 6: 179-185, 2004.

3. Holbourn KP, Acharya KR and Perbal B: The CCN family of proteins: Structure-function relationships. Trends Biochem Sci 33: 461-473, 2008.
4. Perbal B: NOV (nephroblastoma overexpressed) and the CCN family of genes: Structural and functional issues. Mol Pathol 54: 57-79, 2001

5. Pennica D, Swanson TA, Welsh JW, Roy MA, Lawrence DA, Lee J, Brush J, Taneyhill LA, Deuel B, Lew M, et al: WISP genes are members of the connective tissue growth factor family that are up-regulated in wnt-1-transformed cells and aberrantly expressed in human colon tumors. Proc Natl Acad Sci USA 95: 14717-14722, 1998

6. Calvisi DF, Conner EA, Ladu S, Lemmer ER, Factor VM and Thorgeirsson SS: Activation of the canonical Wnt/beta-catenin pathway confers growth advantages in c-Myc/E2F1 transgenic mouse model of liver cancer. J Hepatol 42: 842-849, 2005.

7. Margalit O, Eisenbach L, Amariglio N, Kaminski N, Harmelin A, Pfeffer R, Shohat M, Rechavi G and Berger R: Overexpression of a set of genes, including WISP-1, common to pulmonary metastases of both mouse D122 Lewis lung carcinoma and B16-F10.9 melanoma cell lines. Br J Cancer 89: 314-319, 2003.

8. Xie D, Nakachi K, Wang H, Elashoff R and Koeffler HP: Elevated levels of connective tissue growth factor, WISP-1, and CYR61 in primary breast cancers associated with more advanced features. Cancer Res 61: 8917-8923, 2001.

9. Konigshoff M, Kramer M, Balsara N, Wilhelm J, Amarie OV, John A, Rose F, Fink L, Seeger W, Schaefer L, et al: WNT1-inducible signaling protein-1 mediates pulmonary fibrosis in mice and is upregulated in humans with idiopathic pulmonary fibrosis. J Clin Invest 119: 772-787, 2009.

10. Tian C, Zhou ZG, Meng WJ, Sun XF, Yu YY, Li L, Luo HZ, Yang L, Zhou B and Gu J: Overexpression of connective tissue growth factor WISP-1 in Chinese primary rectal cancer patients. World J Gastroenterol 13: 3878-3882, 2007.

11. Edge SB, Byrd DR, Compton CC, Fritz AG, Greene FL and Tritti A: American joint committee on cancer-cancer staging manual. 7th edition. New York: Springer, 2010.

12. Myung DS, Park YL, Chung CY, Park HC, Kim JS, Cho SB, Lee WS, Lee KH, Lee JH and Joo YE: Expression of Livin in colorectal cancer and its relationship to tumor cell behavior and prognosis. PLoS One 8: e73262, 2013.

13. Nagai Y, Watanabe M, Ishikawa S, Karashima R, Kurashige J, Iwagami S, Iwatsuki M, Baba Y, Imamura Y, Hayashi N and Baba H: Clinical significance of Wnt-induced secreted protein-1 (WISP-1/CCN4) in esophageal squamous cell carcinoma. Anticancer Res 31: 991-997, 2011.

14. Tang Q, Jiang X, Li H, Lin Z, Zhou X, Luo X, Liu L and Chen G: Expression and prognostic value of WISP-1 in patients with endometrial adenocarcinoma. J Obstet Gynaecol Res 37: 606-612, 2011.

15. Chen PP, Li WJ, Wang Y, Zhao S, Li DY, Feng LY, Shi XL, Koeffler HP, Tong XJ and Xie D: Expression of Cyr61, CTGF, and WISP-1 correlates with clinical features of lung cancer. PLoS One 2: e534, 2007.

16. Chuang JY, Chang AC, Chiang IP, Tsai MH and Tang CH: Apoptosis signal-regulating kinase 1 is involved in wisp-1-promoted cell motility in human oral squamous cell carcinoma cells. PLoS One 8: e78022, 2013.

17. Igney FH and Krammer PH: Death and anti-death: Tumour resistance to apoptosis. Nat Rev Cancer 2: 277-288, 2002.

18. Jung J, Cho NH, Kim J, Choi EC, Lee SY, Byeon HK, Park YM, Yang WS and Kim SH: Significant invasion depth of early oral tongue cancer originated from the lateral border to predict regional metastases and prognosis. Int J Oral Maxillofac Surg 38: 653-660, 2009.

19. You Z, Saims D, Chen S, Zhang Z, Guttridge DC, Guan KL, MacDougald OA, Brown AM, Evan G, Kitajewski J and Wang CY: Wnt signaling promotes oncogenic transformation by inhibiting c-Myc-induced apoptosis. Cell Biol 157: 429-440, 2002.

20. Su F, Overholtzer M, Besser D and Levine AJ: WISP-1 attenuates p53-mediated apoptosis in response to DNA damage through activation of the Akt kinase. Genes Dev 16: 46-57, 2002. 